\title{
The spindle assembly checkpoint and speciation
}

\author{
Robert C Jackson ${ }^{1}$, Hitesh B Mistry ${ }^{\text {Corresp. } 2}$ \\ 1 Pharmacometrics Ltd., Cambridge, United Kingdom \\ 2 Division of Pharmacy, University of Manchester, Manchester, United Kingdom \\ Corresponding Author: Hitesh B Mistry \\ Email address: hitesh.mistry@manchester.ac.uk
}

A mechanism is proposed by which speciation may occur without the need to postulate geographical isolation of the diverging populations. Closely related species that occupy overlapping or adjacent ecological niches often have an almost identical genome but differ by chromosomal rearrangements that result in reproductive isolation. The mitotic spindle assembly checkpoint normally functions to prevent gametes with non-identical karyotypes from forming viable zygotes. Unless gametes from two individuals happen to undergo the same chromosomal rearrangement at the same place and time, a most improbable situation, there has been no satisfactory explanation of how such rearrangements can propagate. Consideration of the dynamics of the spindle assembly checkpoint suggest that chromosomal fission or fusion events may occur that allow formation of viable heterozygotes between the rearranged and parental karyotypes, albeit with decreased fertility. Evolutionary dynamics calculations suggest that if the resulting heterozygous organisms have a selective advantage in an adjoining or overlapping ecological niche from that of the parental strain, despite the reproductive disadvantage of the population carrying the altered karyotype, it may accumulate sufficiently that homozygotes begin to emerge. At this point the reproductive disadvantage of the rearranged karyotype disappears, and a single population has been replaced by two populations that are partially reproductively isolated. This definition of species as populations that differ from other, closely related, species by karyotypic changes is consistent with the classical definition of a species as a population that is capable of interbreeding to produce fertile progeny. Even modest degrees of reproductive impairment of heterozygotes between two related populations may lead to speciation by this mechanism, and geographical isolation is not necessary for the process. 


\title{
The Spindle Assembly Checkpoint and Speciation
}

3

4

5

6

\author{
Robert C. Jackson ${ }^{1}$, Hitesh B. Mistry²
}

1 Pharmacometrics Ltd, Cambridge, United Kingdom

2 Division of Pharmacy, University of Manchester, United Kingdom

Corresponding Author

Hitesh Mistry

Street Address: Division of Pharmacy, Manchester, M13 9PT, UK

Email address: hitesh.mistry@manchester.ac.uk

\section{Abstract}

A mechanism is proposed by which speciation may occur without the need to postulate geographical isolation of the diverging populations. Closely related species that occupy overlapping or adjacent ecological niches often have an almost identical genome but differ by chromosomal rearrangements that result in reproductive isolation. The mitotic spindle assembly checkpoint normally functions to prevent gametes with non-identical karyotypes from forming viable zygotes. Unless gametes from two individuals happen to undergo the same chromosomal rearrangement at the same place and time, a most improbable situation, there has been no satisfactory explanation of how such rearrangements can propagate. Consideration of the dynamics of the spindle assembly checkpoint suggest that chromosomal fission or fusion events may occur that allow formation of viable heterozygotes between the rearranged and parental karyotypes, albeit with decreased fertility. Evolutionary dynamics calculations suggest that if the resulting heterozygous organisms have a selective advantage in an adjoining or overlapping ecological niche from that of the parental strain, despite the reproductive disadvantage of the population carrying the altered karyotype, it may accumulate sufficiently that homozygotes begin to emerge. At this point the reproductive disadvantage of the rearranged karyotype disappears, and a single population has been replaced by two populations that are partially reproductively isolated. This definition of species as populations that differ from other, closely related, species by karyotypic changes is consistent with the classical definition of a species as a population that is capable of interbreeding to produce fertile progeny. Even modest degrees of reproductive impairment of heterozygotes between two related populations may lead to speciation by this mechanism, and geographical isolation is not necessary for the process.

\section{Introduction}

The classical definition of a eukaryotic species is a population that is able to interbreed and produce fertile offspring. The ability to interbreed requires that sister chromatids of the two parents are able to align in the process of meiosis, and following recombination, segregate into daughter cells that each contain one and only one member of each pair of replicated chromosomes. Because the recombination process is not $100 \%$ faithful, mis-joining occasionally occurs, with resulting chromosomal rearrangement. The rearranged chromatids are usually unable to align with their unchanged sister chromatids in a way that will produce gametes with a 
46

47

48

49

50

51

52

53

54

55

56

57

58

59

60

61

62

63

64

65

66

67

68

69

72

73

74

75

76

77

78

79

80

81

82

83

84

85

86

87

88

89

90

91

correct gene complement.

Since even closely related species usually differ in their karyotype, it has long been argued that chromosomal rearrangement may lead to speciation [1-6]. The difficulty in this explanation of speciation is that when a chromosomal rearrangement arises in a single individual, if that individual mates with an unchanged partner their unmatched karyotypes will not produce viable zygotes. If enough individuals in a population generate the same chromosomal rearrangements in the same time and place, they could in principle form a new population, reproductively compatible with each other, though not with their ancestral population, and this would constitute a new species [7,8]. However, the probability of this happening seems too low to explain observed rates of evolution.

The process of chromosome segregation during mitosis and meiosis is monitored by the spindle assembly checkpoint (SAC) [9,10]. This assures that daughter cells each receive one copy of each replicated chromosome. It has been argued that the SAC must be involved in speciation $[11,12]$, but at the time these papers were written SAC dynamics were not well understood, and the proposed mechanism did not answer the question of how the rearranged karyotypes were able to propagate. In recent years the SAC has been an object of intensive study, and its dynamics are increasingly well understood [10,13-21]. The details of the SAC have been most extensively studied in mitosis. There are differences between mitosis and meiosis I (co-orientation) and meiosis II (bi-orientation) which have been reviewed by Ohkura [22]. Our focus in the present discussion is on meiosis II, and we make the assumption that tension signalling is similar to that in mitosis.

Central to the operation of the SAC is the generation of a "wait" signal which suppresses progression to anaphase until all kinetochores are correctly attached by microtubules to the spindle poles. At this point, the wait signal rapidly decays, and the anaphase promoting complex/cyclosome (APC/C), released from inhibition, dissolves the cohesin links between sister chromatids, and the cell proceeds to anaphase. Several kinds of rearrangement are possible: in the following discussion we focus on fission, in which a single chromosome is broken in two, or fusion, in which two chromosomes that should be separate become joined together. A consideration of our present understanding of SAC dynamics shows how, in certain circumstances, chromosomas fission or fusion can lead to speciation.

\section{Normal operation of the spindle assembly checkpoint}

The SAC acts by sensing correct connections of kinetochores to the two opposite spindle poles. Until all kinetochores are attached, and in a state of tension, a "wait" signal is released that prevents progression to anaphase (figure 1). The wait signal is mediated by a complex group of microtubule-associated proteins, constituting the mitotic checkpoint complex (MCC) [9]. Of these, the most upstream is a bistable, tension-sensitive enzyme, believed in insects to be aurora, or in vertebrates the homologous aurora kinase B [16]. Either over-expression of aurora kinase $\mathrm{B}$, or its inhibition in mammalian cells results in aneuploidy [23,24]. Microtubules grow from the opposite spindle poles, and attach randomly to the kinetochores (in fact each kinetochore has 20 - 30 microtubule binding sites). When the kinetochore of one of a pair of sister chromatids is attached to a single spindle pole, the situation described as "monotelic" in figure 1, the 
92 kinetochore is not in a state of tension. Now when a microtubule attaches to the other sister

93 chromatid, we assume that there is a $50 \%$ chance that it will be attached to the opposite spindle

94 pole (amphitelic attachment), and a 50\% chance that it will attach to the same spindle pole as its

95 sister (syntelic attachment). Syntelic attachments are not in a state of tension, which results in

96 the second attachment being removed through activity of the aurora enzyme [10,13-15]. After

97 this, there is again a 50:50 chance that the second kinetochore will become correctly attached.

98 Amphitelic attachments are in a state of tension, aurora kinase B is inactive, so that a particular

99 sister chromatid pair does not produce a wait signal. When the last pair of sister chromatids in

100 the cell is correctly attached, the wait signal rapidly decays, and the cell progresses to anaphase,

101

102

103 In addition to monotelic, syntelic, and amphitelic attachments, a fourth state is possible, 104 merotelic attachment, in which one kinetochore is correctly attached while the other is 105 simultaneously bound to microtubules from both spindle poles. Merotelic attachments do not 106 appear to be detected by the SAC, and are likely to lead to abnormal mitoses or meioses. For the 107 purpose of our analysis, we assume that the frequency of merotelic attachments is the same for 108 cells with normal or rearranged karyotypes, in which case they would not influence the relative incidence of zygotes with a normal gene complement.

\section{Operation of the SAC following chromosomal fission or fusion}

Now consider the situation discussed by Kolnicki [11], in which kinetochore duplication has resulted in a particular chromosome being split into a pair of smaller, acrocentric chromosomes. Figure 2 describes the events in metaphase II of meiosis in this cell. Any syntelic attachments formed prior to the attachment of the second acrocentric will be reversed. At the stage where the single un-fissioned chromatid and one of the acrocentrics have become amphitelically attached,

118 two possible configurations (or their mirror images) will result. These are shown as A and B in figure 2. For each of these configurations, there is a $50 \%$ chance that the remaining unattached kinetochore will become correctly attached, resulting in configuration $\mathrm{C}$. Of the remaining 50\%, $25 \%$ will attach to give configuration D, and $25 \%$ will give configuration E. Systems C, D, and E are all in tension, and will progress to anaphase. Configuration $\mathrm{C}$ will yield equal numbers of gametes with the karyotypes shown as type I and type II in figure 3. Configuration D will yield equal numbers of the gametes labelled type III and type IV. Configuration E will result in equal numbers of gametes type V and type VI (figure 3). Gametes of type III and type V are missing many genes, and will probably be nonviable. Gametes of type IV and type VI contain a complete complement of genes, and may progress to fertilisation.

When fertilisation is by wild-type gametes, the possible resulting zygotes are as illustrated in figure 4. Fertilisation of gametes of type I gives wild-type zygotes (A in figure 4). Fertilisation of type II gametes gives zygotes that are heterozygous for the chromosomal fission (fig 4B). These contain a full gene complement, and normal gene dosage. These zygotes are expected to be fully viable, and their fate will be considered below. Gametes of types IV and VI, after fertilisation by a wild-type gamete, will give zygotes of types $C$ and )D. These have abnormal gene dosage, and are likely either to spontaneously abort, or to develop into offspring with birth defects. These surviving individuals will not necessarily be sterile, but are likely to have at least partially decreased fertility. 
138

139

140

141

142

143

144

145

146

147

148

149

150

151

152

153

154

155

156

157

158

159

160

161

162

163

164

165

166

167

168

169

170

171

172

173

174

175

176

177

178

179

180

181

182

183

In summary, when neokinetochore formation, and subsequent chromosomal fission, occur in meiosis $25 \%$ of the resulting gametes will receive the normal monocentric chromatid. Of the remaining $75 \%$, one-third result in gametes with a pair of acrocentric chromosomes. These cells will be stable, and segregate correctly at mitosis. The other $50 \%$ will give half (25\% overall) gametes with missing genes and half (25\%) gametes with incorrect gene dosage. If neokinetochore formation occurs without chromosomal fission, the result will be $50 \%$ of gametes with a bicentric chromosome. For the reasons discussed by Kolnicki [11], these chromosomes will have a $50 \%$ chance of splitting into two acrocentrics at each subsequent mitosis. The overall effect on fertility will be the same as the situation discussed above, in which chromosomal fission immediately follows neokinetochore formation.

A similar analysis may be performed for the situation where two chromosomes fuse. If the fused chromosome is capable of aligning during meiosis with the two corresponding wild-type chromosomes, then viable and non-viable gametes will result in the ratios discussed for chromosomal fission. Obviously if the fused chromosome is incapable of alignment with its cognate parental chromosomes, the chromosomal rearrangement will be lethal.

\section{Methods}

The speciation algorithm is a form of genetic algorithm [25,26] (figure S1, supplementary material). A growth equation calculates reproduction rates for the various karyotypes. The objective function determines how many individuals of each karyotype survive to reproductive age in the next generation, and also how many individuals migrate between ecological niches. Positive feedback from the objective function to the growth equation indicates that those individuals best adapted to their environment represent a higher proportion of the next generation. Information on fertility of karyotypic variants is reflected in growth rates used by the growth equation; for heterozygotes, fertility rates following chromosomal re-arrangements (including chromosomal fusion, fission, and inversions) will be lower than for homozygotes. Information on the degree of adaptation of variants to their environment is included in the objective function. The model simulates populations in two overlapping or adjoining ecological niches, and individuals can move between the two niches. The calculations discussed below consider a hypothetical rapidly reproducing organism with a potential population doubling time of 10 weeks.

Chromosome fusion or fission will result in only 50\% of gametes having correct chromosome segregation in meiosis, with the other $50 \%$ having potentially lethal abnormalities in gene dosage (figure 4). The overall fertility of heterozygotes is thus assumed to be reduced by up to $50 \%$. Offspring homozygous for chromosomal rearrangements are assumed to have unimpaired fertility. Initial simulations treated population growth as exponential, until the carrying capacity of the environment was reached, after which the net growth rate became zero.

Programming. The speciation algorithm has been implemented in a computer program written in the $\mathrm{R}$ language. Source code is included in the supplementary material and can be found here: https://github.com/HiteshBMistry/Speciation. Graphics were generated using R graphics, or the open source program, gnuplot [27].

Peer) reviewing PDF | (2019:11:43037:1:1:NEW 6 Mar 2020) 


\section{Results}

186

\section{Effects of chromosome fusion in a species occupying a single ecological niche.}

Initially, to explore the population dynamics, we assume a small pre-existing fraction of the karyotypic variants in the population, without at this stage inquiring how they got there. Simulation 1 (Table 1) assumed that birth and death rates for individuals carrying the additional kinetochore were unchanged from those of the original population (shown as Z1 in the tables and figures). However, as discussed in figure 4, the efficiency of formation of viable gametes will be reduced by $50 \%$ in the heterozygous variant population, Z2. Reproductive efficiency of the homozygous variant population, Z3, is unimpaired. Figure 5 shows that the heterozygous variant population, Z2, increased at half the rate of the original population in the early stage of the growth curve. It was assumed that death rates for all populations remained unchanged until the carrying capacity of the environment (the asymptotic population, AP) was reached, at which point the death rate increased abruptly to equal the birth rate. Alternative growth curves are explored later. Heterozygotes (Z2) as a fraction of the total population declined from the outset. When the carrying capacity of the environment, AP, was reached (at 39 days in table 1; call this the asymptotic time, AT) the total population levelled off. Z2 then declined in absolute numbers. Homozygous variants (Z3) declined in absolute numbers from the outset (if, as seems unlikely, Z3 was ever nonzero: nonintegral values of population sizes may be interpreted as probabilities). After AT the rate of decline of Z3 increased. Z2 fell below 0.5 at 220 days. This will be referred to as the extinction time, ET.

Simulation 2 explores the effect of founder population size. The population of Z1 is now 2 logs smaller than it was in table 1 , and the combined initial population of homozygous and heterozygous variants $(\mathrm{Z} 2+\mathrm{Z3})$ is now equal to $\mathrm{Z} 1$. However, the variants still became extinct, though it took longer. If the initial population of homozygous chromosomal variants, Z3, was larger than the wild-type population, then the variants became the dominant population, and the wild-type moved towards extinction (simulation 3). Simulation 4 shows the extreme case where the initial numbers of $\mathrm{Z} 1, \mathrm{Z} 2$, and $\mathrm{Z3}$ were identical. In this case the variant did not become extinct, and the ratio of the three karyotypes in the asymptotic population was the Mendelian $1: 2: 1$. This state, in which wild-type and variant maintain equal populations, is unstable: a transient decrease in either of the homozygous populations will result in its eventual extinction. Note that changing the initial number of heterozygotes did not change the outcome, except by increasing the time to the asymptote (simulation 5). To summarize simulations $1-5$ of Table 1 : when the growth parameters (doubling time and death rate) for wild-type and variant are identical, whichever has the smallest population will eventually become extinct. The reason for this is that the heterozygous population has equal

numbers of wild-type and variant chromosomes, and thus a greater proportion of whichever population is the smaller. Because the heterozygotes have lower fertility, this results in an everdecreasing proportion of whichever chromosome type starts out with the smaller population. In a situation where the chromosomal variant results from a spontaneous re-arrangement, the variants will always be outnumbered, so that, other things being equal, variants will always become extinct. This is how the SAC maintains karyotypic stability. 
230

231

232

233

234

235

236

237

238

239

240

241

242

243

244

245

246

247

248

249

250

251

252

253

254

255

256

257

258

259

260

261

262

263

264

265

266

267

268

269

270

271

272

273

274

275

Even a slight population disadvantage of the variants, however, resulted in their extinction (table 1, simulation 6 and table S1, supplementary material). Simulation 7 assumed that the death rate for all populations was increased by a factor of 5 . This resulted in $>3$-fold increase in time to asymptote, but time to extinction of the variant population was unchanged. Simulation 8 made the more conservative assumption that the heterozygotes had only a slight fertility disadvantage, with a potential population doubling time of 10.5 weeks, only $5 \%$ longer than normal. The variants still became extinct, though it took much longer. Increasing the death rate to 0.07/ week or higher resulted in extinction of the entire population (table S1). Decreasing all death rates to zero decreased the time to asymptote to 33.5 weeks, but time to extinction of the variants was unaffected (simulation 9). Increasing the death rate to $0.07 /$ wk resulted in extinction of the entire population (simulation 10).

Simulation 11 (table 1) now assumed that the karyotypic variant carried a dominant mutation that conferred a survival advantage in the original ecological niche. Time to asymptote was slightly decreased, and the variant now dominated the entire population, with the wild-type eventually becoming extinct. If the wild-type population originally existed in two or more geographically separated environments, then the variant, which is now both reproductively and geographically isolated from the wild-type, will have become a new species. This would be an example of allopatric speciation. Simulation 12 assumed the same parameter values as simulation 11, except that the mutation in the karyotypic variant strain was now treated as recessive. In this case, the variant became rapidly extinct. Returning to the assumption that the mutation carried by the variant was dominant, the degree of advantage conferred by the mutation was important. In simulation 13, the decrease in death rate caused by the mutation was less than 3-fold, and the variant still became extinct. Decreasing the death rate of the homozygous and heterozygous variants slightly more, to 0.016 , was sufficient to tip the balance in favour of the variant strain (simulation 14).

In practice, chromosomal variants must first present as a single heterozygous individual. Simulation 11 models that situation, and shows that, given a survival advantage sufficient to offset their reproductive disadvantage, the progeny of that single individual will eventually become the majority population.

It may be argued that the growth curve used in the previous simulations was overly simplistic. Growth was assumed to be exponential until the carrying capacity of the ecosystem was reached, at which point growth abruptly became zero. A more realistic scenario can be modelled using a form of the Gompertz equation (table 2, line 2). Population increase was assumed to reflect a balance between birth and death. Birth rates were treated as constant, but death rates were treated as inversely proportional to the log of resource availability, defined as log distance from the carrying capacity, AP. For a population of 1, the death rate is zero; at the carrying capacity the death rate is a steady-state value, $\mathrm{DR}_{\mathrm{ss}}$, (equal to the birth rate) and at intermediate populations, $\mathrm{Z}$, the death rate $=\mathrm{DRss} * \log \mathrm{Z} / \log$ AP. The parental population approached its carrying capacity asymptotically, and the homozygous and heterozygous variants slowly became extinct.

Another growth curve widely used in population studies is the discrete logistic model [28].

Peer] reviewing PDF | (2019:11:43037:1:1:NEW 6 Mar 2020) 
276 Using this growth equation in the speciation algorithm, as with the Gompertzian growth curve,

277 changed the time taken for the two populations to diverge, but did not alter the qualitative

278 behaviour of the system (table 2; line 3). The early part of the normal growth curve (figure 6)

279

280

281

282

283

284

285

286

287

288

289

290

291

292

293

294

295

296

297

298

299

300

301

302

303

304

305

306

307

308

309

310

311

312

313

314

315

316

317

\section{Conclusions from studies with the model of a chromosomal variant in a single ecological niche}

Consideration of spindle assembly checkpoint dynamics indicates that a mismatch in chromosome numbers between maternal and paternal karyotypes will result in formation of $50 \%$ of normal gametes, and 50\% with deleted or duplicated genetic material. These defective gametes, when fertilised, are likely to lead to nonviable zygotes, or zygotes that develop into sterile or partially sterile offspring. Thus, heterozygotes will have fertility that is lower than normal by up to $50 \%$. The degree of reproductive impairment affects the time taken for the variants to become extinct, but does not change the final outcome. This conclusion applies both to chromosomal rearrangement caused by neokinetochore formation (giving an increased chromosome number) and chromosomal fusion (leading to decreased chromosome number). Zygotes that are homozygous for the rearranged chromosomes should segregate normally in mitosis and meiosis, and have unchanged fertility. The qualitative behaviour of the system is independent of the detailed form of the growth curve (exponential, logistic, or Gompertzian). If the variant is assumed to have a significantly lower death rate than wild-type, this may be sufficient to overcome the variant's reproductive disadvantage, and the wild-type may become extinct in its original habitat. For this to happen, the mutation causing the lower death rate must be dominant.

\section{Simulating the effects of chromosome fusion in a species that can populate two overlapping ecological niches.}

Subsequent simulations assumed that the organism under study could survive in either of two ecological niches. These niches could be overlapping or adjacent geographically, but differed in some factor that affected survival - temperature, water availability, availability of food or shelter, presence of predators or parasites. The original strain of the organism is poorly adapted to niche 2 ,

and this was reflected in a higher death rate in this environment. The initial assumption was that no organisms lived in niche 2 , but that when the carrying capacity of niche 1 was reached, organisms colonised niche 2 (e.g. in search of food). Initially this migration was assumed to be one-way. Table 3, simulation 1 assumed that generation times and death rates did not differ between niche 1 and niche 2 . The karyotypic variant became extinct in both niche 1 and niche 2 . The total population in niche 2 was slightly greater than the carrying capacity because of immigration.

Table 3, simulation 2 shows the result of modelling the growth of organisms in the two niches, where Z4 (the original karyotype) had a high death rate in niche 2, and where the carrying capacity of niche 2 was limited. Only 1\% of migrants survived. Two separate populations emerged, but a stable fraction of heterozygotes remained (Figure 7). Does this represent 
322

323

324

325

326

327

328

329

330

331

332

333

334

335

336

337

338

339

340

341

342

343

344

345

346

347

348

349

350

351

352

353

354

355

356

357

358

359

360

361

362

363

364

365

366

367

368

speciation? It does in the sense that two stable, karyotypically distinct populations co-existed in the same overall geographical environment. A small population of partially fertile heterozygotes persisted. This is analogous to the situation seen (e.g.) with big cat species [2].

If we assume that migration into niche 2 ceased after 350 weeks (table 3 , simulation 3 ), by 600 weeks there were no variant type organisms in niche 1 , and no wild-type organisms in niche 2 Depending on our definition, speciation has resulted. The two divergent populations were geographically isolated, so this may be regarded as an instance of allopatric speciation. If we assume that the mutation that accompanied the chromosomal rearrangement was recessive, then speciation did not occur (table 3, simulation 4).

Table 3 simulation 5 is a repeat of table 3 , simulation 2, but now making the assumption that $100 \%$ of members of populations 2 and 3 that migrated to niche 2 survived to reproduce. This did not affect the outcome: the karyotypic variant became the dominant population in niche 2 , though the steady state population balance was achieved more rapidly than when only a small fraction of the immigrants survived. Changing the survival factor for the wild-type to 0.001 , that is, assuming that only one per thousand of migrants into niche 2 survived to reproduce, reduced the number of heterozygotes in niche 2 to $<0.2 \%$ (table 3 , simulation 6 ).

If the process of meiosis is inefficient when a pair of short chromosomes has to align with a single long partner, efficiency of the overall process may be decreased below the $50 \%$ calculated from the spindle checkpoint kinetics. Table 3, simulation 7 assumes that efficiency of the pairing process is reduced by $50 \%$, extending the doubling time of heterozygotes (populations 2 and 5) to 40 weeks. Table 3 , simulation 8 combined inefficient hybridization with low survival factors. This set of parameter values resulted in heterozygotes being decreased to about $0.12 \%$ of the total population. According to this model, they can be decreased to an arbitrarily low level but never eliminated.

Table 4, simulation 1 modelled the effect of using a different expression for migration rates. Previously the assumption had been made that migration only occurred when the total population in niche 1 was above the carrying capacity:

$$
\operatorname{mg}[\mathrm{n}]=\mathrm{XS} * \operatorname{frac}[\mathrm{n}] * \operatorname{sf}[\mathrm{n}]
$$

where $\operatorname{mg}[\mathrm{n}]$ is the number of migrants of karyotype $\mathrm{n}, \mathrm{XS}$ is the excess population above the carrying capacity, frac[n] is the size of population $n$ as a fraction of the total population, and $\mathrm{sf}[\mathrm{n}]$ is the survival factor for karyotype $n$, i.e. the fraction of immigrants of karyotype $\mathrm{n}$ in niche 2 who survived to reproduce. As an alternative, let migration be inversely proportional to the difference between total population and carrying capacity:

$$
\operatorname{mg}[\mathrm{n}]=\mathrm{Z}[\mathrm{n}] * \mathrm{Z}[0] / \mathrm{AP} * \mathrm{sf}[\mathrm{n}]
$$

where $Z[n]$ is the population size of karyotype $n$ in niche $1, Z[0]$ is the total population in niche 1 , AP is the asymptotic population in niche 1 (the carrying capacity), and $\mathrm{mg}[\mathrm{n}]$ and $\mathrm{sf}[\mathrm{n}]$ are as defined above. As before, the karyotypic variant became extinct in niche 1, and became the dominant population in niche 2 , though the proportion of heterozygotes in niche 2 was larger than when migration was only assumed to occur after the population in niche 1 reached its 
369

370

371

372

373

374

375

376

377

378

379

380

381

382

383

384

385

386

387

388

389

390

391

392

393

394

395

396

397

398

399

400

401

402

403

404

405

406

407

408

409

410

411

412

413

414 carrying capacity. This alternative expression for migration rates is used in conjunction with the discrete logistic growth curve. Table S2 (suplementary material) shows an example of a single heterozygous cell in niche 1 giving rise to a nascent species in niche 2 , even though the variants became extinct in niche 1 .

Table 4, simulation 2 shows a repeat of simulation 1, but assumes that migration is bidirectional. This model was used to explore 2-way migration, for example with migration in each direction inversely proportional to food availability (i.e. directly proportional to population) or directly proportional to death rate. Survival factors were set to 1.0 for populations 2 and 3 , and to 1e-6 for population 1. The variants became the dominant population in niche 2 . Subsequent simulations found that the survival factor of the original strain could be as high as $7 \times 10^{-2}$, but values of $8 \times 10^{-2}$ and higher resulted in the variant population becoming extinct in niche 2 (Table S2). In each case the steady state number of heterozygotes in niche 2 , sustained by inward migration of wild-type organisms, was very low, but non-zero. Allowing backmigration had the paradoxical effect of increasing the steady state populations of wild-type (Z4) and heterozygotes (Z5) in the population of niche 2. Comparing simulation 2 with simulation 1 (table 4), this seems to be because the back migration caused a higher steady state population of heterozygotes (Z2) in niche 1 (and to a much lesser extent of the homozygous variants, Z3).

Simulations 4 and 5 of table 4 compare the migration from niche 1 to niche 2 of chromosomal variants associated with a mutation that confers a survival advantage in niche 2 . Even a recessive mutation (simulation 5) was able to establish a population of variant homozygotes in niche 2 , though the number of heterozygotes was much lower than for simulation 4 , where the mutation was dominant.

Table 4, simulations 6 and 7 modelled the situation where both niches were partially occupied before the chromosomal rearrangement occurred. The model showed that a low level of occupancy of niche 2 by the wild-type did not alter the final steady-state distribution, although it took longer to reach (simulation 6). The outcome was dependent on the initial conditions: a small increase in the death rate resulted in the variant population slowly becoming extinct (simulation 7).

\section{Simulating geographical isolation}

Table 3, simulation 3 predicted that if migration from niche 1 to niche 2 was completely stopped after 350 weeks, that only the wild-type strain would survive in niche 1 , and only the variant strain would survive in niche 2. Geographical separation of two populations thus resulted in different variants dominating in the two environments, based upon their different adaption to their respective environments. It may be argued that this in itself does not represent speciation, because there is nothing to stop the two populations subsequently interbreeding and forming partially fertile heterozygotes. The new strain in niche 2 has become, in the terminology of Bush [3], a host race. The present analysis, however, has shown that, in the absence of continued migration from niche 1 , the wild-type organisms and heterozygotes in niche 2 will become extinct.

Figure 8 shows the result of repeating simulation 3 of table 3 , but now allowing migration 
415 from niche 1 to niche 2 to resume, after the original karyotype had become extinct in niche 2. A 416 low level of heterozygotes now re-appeared, and an even lower level of the original homozygous 417 strain. This shows that the continued existence of heterozygotes in niche 2 was dependent upon 418 continued immigration.

419

420

421

422

423

424

425

426

427

428

429

430

431

432

433

434

435

436

437

438

439

440

441

442

443

444

445

446

447

448

449

450

451

452

453

454

455

456

457

458

459

460

\section{Discussion}

Inaccurate chromosome segregation in germ line cells must confer a selective disadvantage. If it were otherwise, stable species could not exist. For a karyotypic variant to diverge from the ancestral line requires either a compensating environmental advantage (which could be conferred by absence of the ancestral line, as occurs in allopatric speciation) or a genetic advantage. Dawkins [29,30] presents the current consensus view of speciation as being driven primarily by geographic separation. He argues that "gene flow but not much" is necessary between habitats [31]. Clearly some gene flow is required for additional habitat to be colonized, but if the level is too high, the two populations will be sufficiently mixed that they will not diverge. Simulations in the present study support this conclusion. On p. 320 of Dawkins [31] he makes the statement "it isn't always a geographic separation". If an adjoining or overlapping niche is hostile to the wild-type organism, but less so to the variant, reproductive isolation may occur despite close geographical proximity (pp. 423-424 of reference 7). Again, the present modelling study has shown that reproductive isolation, even if incomplete, can drive peripatric speciation. Our studies support the conclusion that geographical isolation is not necessary for speciation, though it may be an enabling factor. Reproductive isolation, however, is both necessary and sufficient.

The conclusions that chromosomal rearrangement in meiosis is a primary driver of speciation, and that the dynamics of the SAC provide the main mechanism of those rearrangements, rest upon the following premises:

Pairs of acrocentric chromosomes resulting from neokinetochore formation are capable of meiotic pairing, with at least partial efficiency, with the cognate mediocentric chromosome from which they were derived. Alternatively, a single chromosome resulting from chromosome fusion (with subsequent loss of one kinetochore) is capable of meiotic pairing, with at least partial efficiency, with the cognate chromosomes from which it was derived.

The SAC in such pairings will delay anaphase until one kinetochore in each chromatid is correctly attached. If, in the chromatid with two kinetochores, one is incorrectly attached, the SAC will still register tension, and such cells may proceed to anaphase.

$50 \%$ of gametes resulting from such pairings will have a full genetic complement, and will be fully fertile. $25 \%$ of gametes will lack part of the genome, and are likely to be infertile. The remaining $25 \%$ of gametes will have part of the genome duplicated, resulting in abnormal gene dosage. This may result in spontaneous abortion or in birth defects. The overall fertility of heterozygotes between wild-type germ cells and those resulting from the two specific kinds of chromosomal rearrangements discussed in (1) will thus be $>50 \%<100 \%$. This reduced fertility will result in the chromosomal rearrangement being eventually eliminated by natural selection, even though the fertility of homozygotes carrying the rearrangement is unimpaired.

If the rearranged chromosome resulting from chromosomal fusion, or one of the acrocentric chromosomes resulting from neokinetochore formation carries a mutation that enhances 
survival, this survival advantage may be sufficient to counter the decreased fertility of heterozygotes. Once sufficient numbers of rearranged homozygotes appear, since they do not have decreased fertility, they will be favoured by selection and become the dominant population, while remaining the same species.

If the rearranged chromosome resulting from chromosomal fusion, or one of the acrocentric chromosomes resulting from neokinetochore formation carries a mutation that enhances survival in a different, but accessible, ecological niche, the possibility exists that the variant will become the dominant population in that second niche, while the wild-type remains the dominant population in the original habitat. The two populations, while occupying overlapping or adjacent geographical territories, have different chromosome numbers, are partially reproductively isolated, and may now be considered different species.

It is possible that chromosomal rearrangements other than fission or fusion could result in decreased fertility, and resulting partial reproductive isolation of the variants, and that this could lead to peripatric speciation. However, only fissions and fusions that result in partial reproductive isolation determined by the dynamics of the SAC, as discussed above, will result in pairs of related species whose karyotype differs in number by a single chromosome pair.

These qualitative conclusions are independent of the equations used to describe population growth or the migration process.

Modelling such a complex organelle as the SAC involves simplifying assumptions. In fact, both tension and attachment are signalled, but our analysis of the effects of chromosomal fission or fusion has focussed upon tension. Configurations A and B of figure 2 are under tension, and according to our model would proceed to anaphase. However, they contain unattached kinetochores that may continue to produce a wait signal. The effect of this would be to produce fewer gametes of type IV and VI than indicated in figure 3, and following fertilization, fewer zygotes of type (3) and (4) as shown in figure 4. The model may thus over-estimate the effect of chromosomal fusion and fission on fertility. We have repeated our calculations assuming that the fertility of heterozygotes is decreased by less than $50 \%$. In each case, the qualitative outcome (extinction or speciation) was the same, though the time course was different. For example, if a population of 10,000 individuals with a doubling time of 10 weeks contained a single heterozygote resulting from chromosomal fusion and the fertility deficit was $50 \%$, so the variant doubling time was 20 weeks, the variant became extinct in 20.5 weeks. If we instead assumed only a $20 \%$ decrease in fertility of heterozygoes, resulting in doubling time of 12.5 weeks, the variants were extinct at 50.5 weeks.

It may be objected that this mechanism of speciation requires the unlikely simultaneous occurrence of two events: a chromosomal rearrangement, and a germ-line mutation. In fact, chromosomal rearrangements during meiosis are not particularly rare. It is estimated that $20 \%$ of all human pregnancies spontaneously abort, and it is likely that a high proportion of these are accounted for by karyotypic abnormalities [32]. In any event, speciation events are very rare, and do not appear to be out of line with the frequency predicted by the present analysis. A possibility not considered by our model is that chromosomal rearrangements could sometimes provide a selective advantage (or disadvantage) without the involvement of a mutation on the rearranged chromosome; this could happen, for example, if a gene were brought by the 
507

508

509

510

511

512

513

514

515

516

517

518

519

520

521

522

523

524

525

526

527

528

529

530

531

532

533

534

535

536

537

538

539

540

541

542

543

544

545

546

547

548

549

550

551

552

rearrangement under the control of a different promoter.

The suggestion that chromosomal rearrangement is a primary driver of speciation has a long history [6]. Dobzhansky, in particular, argued that different chromosomal numbers in each parent would interfere with meiosis, causing sterility of the hybrids [5]. He argued that chromosomal rearrangement, allelic mutations, environmental changes, and isolation of populations all contributed to speciation. The reaction of Dobzhansky's contemporaries, and the role of mathematical modelling in assessing competing hypotheses of speciation, have been critically reviewed by Schwartz [33]. The principal objection to chromosomal rearrangement as a driver of speciation has been that such rearrangements result in reproductive incompatibility with the ancestral karyotype, so it is difficult to see how they can be propagated. Parris $[7,8]$ has argued that the probability of simultaneous identical rearrangements may sometimes be sufficient to allow these "hopeful monsters" to find a mate. The present model, based upon the partial fertility of heterozygotes, largely supports Dobzhansky's main conclusions, but carries the argument forward by showing that a consideration of the dynamics of the SAC is necessary to build a consistent model that incorporates these various contributions to speciation.

Despite persistent suggestions [34-37] that Darwin did not explain the origin of species, the present analysis is consistent with natural selection being a primary driving force of speciation. The essence of Darwinism is that competition for resources between related variants is determined by an objective function (natural selection). When the related variants are germ-line mutations or recombinants resulting from crossing-over during meiosis, natural selection results in adaptation of a species to its environment. When the variants are the result of chromosomal rearrangements with resulting partial reproductive isolation, the result is speciation.

The function of the SAC is to ensure descent without modification. The mechanism by which it achieves this is complex and error-prone. It deals with errors by making them lethal, and therefore irrelevant. Most of the time - sometimes for millions of generations - this works very well, and ensures the integrity of the species. Most of the time, genetics can focus on the genes present in the genome, without being concerned with how they are packaged. Once in a great while, this quality control process is undermined by a simultaneous, compensating error that allows heterozygotes expressing a small sub-set of chromosomal rearrangements to persist and multiply. The result is two stable, reproductively isolated populations (species) with different karyotypes. The dynamics of the SAC have been selected to maintain stable karyotypes. It is the rare events that subvert this process that explain the origin of species and make it possible for evolution of sexually reproducing species to occur.

Other computational models of speciation have emphasised the importance of gene flow [3842]. Our explanation of speciation differs from earlier models in placing the emphasis on reproductive isolation caused by chromosomal changes. We do not discount the role of gene flow: indeed, our model shows that karyotypic changes can only propagate in the presence of genetic mutations that confer a sufficient survival advantage to counter the inherent reproductive disadvantage of the chromosomal rearrangements.

Based on the interpretation of speciation discussed here, the critical difference between humans and the great apes is not brain size, or bipedal gait, or the use of tools. It is the fact that 
553 humans have 23 pairs of chromosomes, and the apes, with an almost identical complement of 554 genes, have 24 pairs. The difference resulted from fusion of the ape chromosomes $2 \mathrm{a}$ and $2 \mathrm{~b}$ $555[43,44]$. The first hominin was the first individual to have that karyotype and pass it on to fertile 556 offspring. This could only have happened if the chromosome fusion was accompanied by a mutation that was able to counter that chromosomal rearrangement's reproductive disadvantage. What was that mutation?

559

560

561

562

563

564

565

566

567

568

569

570

571

572

573

574

575

576

577

578

579

580

581

582

583

584

585

586

587

588

589

590

591

592

593

594

595

596

597

598

\section{References}

1. Mayr E (1970) "Populations, Species, and Evolution". Cambridge MA. Belknap Press of Harvard University Press (1970).

2. Wilson EO (2001). "The Diversity of Life", new edition, pp. 47-68. London. Penguin Books.

3. Bush GL (1994). Sympatric speciation in animals: new wine in old bottles. Trends Ecol Evol 9: 285-288.

4. Todd NB (1970), Karyotypic fissioning and canid phylogeny. J Theoret Biol 26: 445480.

5. Dobzhansky T (1941). "Genetics and the Origin of Species", $2^{\text {nd }}$ Edition, New York: Columbia University Press.

6. Ruffié J (1986) The Population Alternative: A New Look at Competition and the Species (translated by Garey L). New York, Pantheon Books.

7. Parris GE (2011) The hopeful monster finds a mate and founds a new species. Hypoth Life Sci 1: 32-37.

8. Parris DE (2013) Application of a hypothesis to speciation in hominidae. Hypoth Life Sci 3: 2-5.

9. Musacchio A, Salmon ED (2007). The spindle assembly checkpoint in space and time. Nature Rev Mol Cell Biol 8: 379-393.

10. Sear RP, Howard M (2006). Modeling dual pathways for the metazoan spindle assembly checkpoint. Proc Natl Acad Sci USA 103: 16758-16763. 
599

600

601

602

603

604

605

606

607

608

609

610

611

612

613

614

615

616

617

618

619

620

621

622

623

624

625

626

627

628

629

630

631

632

633

634

635

636

637

638

639

640

641

642

643

644

11. Kolnicki RL (2000), Kinetochore reproduction in animal evolution: cell biological explanation of karyotypic fission theory. Proc Natl Acad Sci USA 97: 9493-9497.

12. Margulis L, Sagan D (2002). "Acquiring Genomes. A Theory of the Origins of Species". pp. 191-199. New York, Basic Books.

13. Kops GB, Weaver BA, Cleveland DW (2005). On the road to cancer: aneuploidy and the mitotic checkpoint. Nat Rev Cancer 5: 773-785.

14. Mistry HB, MacCallum DE, Jackson RC, Chaplain MAJ, Davidson FA (2008). Modeling the temporal evolution of the spindle assembly checkpoint and role of Aurora B kinase. Proc Natl Acad Sci USA 105: 20215-20220.

15. Mistry HB, MacCallum DE, Jackson RC, Chaplain MAJ, Davidson FA (2010). A pharmacodynamic model of aurora kinase inhibitors in the spindle assembly checkpoint. Frontiers in Bioscience 15: 249-258.

16. Campbell CS, Desai A (2013) Tension sensing by aurora B kinase is independent of survivin-based centromere localization. Nature 497: 118-121.

17. Sarangapani KK, Asbury CL (2014) Catch and release: how do kinetochores hook the right microtubules during mitosis? Trends Genet 30: 150-159.

18. Britigan EM, Wan J, Zasadil LM et al. (2014) The ARF tumor suppressor prevents chromosome instability and ensures mitotic checkpoint fidelity through regulation of Aurora B. Mol Cell Biol Jul 23. pii: mbc.E14-05-0966.

19. Peplowska K, Wallek AU, Storchova Z (2014) Sgol regulates both condensing and Ipl1/Aurora B to promote chromosome biorientation. PLoS Genet 10: e1004411. doi: 10.1371/journal.pgen 1004411.

20. Kim S, Meyer R, Chuong H, Dawson DS (2013) Dual mechanisms prevent premature chromosome segregation during meiosis. Genes Dev 27: 2139-2146.

21. Proudfoot KG, Anderson SJ, Dave S, Bunning AR, Roy PS, Bera A, Gupta ML (2019) Checkpoint proteins Bub1 and Bub3 delay anaphase onset in response to low tension independent of microtubule-kinetochore detachment. Cell Rep 27: 416-428.

22. Ohkura H. Meiosis: an overview of key differences from mitosis. Cold Spring Harb Perspect Biol 2015 Jan 20 7(5). pii: a015859. doi: 10. 1101/cshperspect.a01589.

23. Gonzalez-Loyola A, Fernandez-Miranda G, Trakala M, Partida D, Samejima K, Ogawa H, Canamero M, Martino A, Martinez-Ramirez A, Carcer G, Castro IP, Earnshaw W, Malumbres M (2015) Aurora B overexpression causes aneuploidy and p21Cip1 repression during tumor development. Mol Cell Biol 35: 3566-78.

24. S Wang, CA Midgley, F Scaërou, JB Grabarek, Griffiths G, Jackson W, Kontopidis G, McClue S, McInnes C, Meades C, Mezna M, Plater A, Stuart I, Thomas MP, Wood G, Clarke RG, Blake DG, Zheleva DI, Lane DP, Jackson RC, Glover DM, Fischer PM (2010) Discovery of N-phenyl-4-thiazol-5-yl) pyrimidine-2-amine aurora kinase inhibitors. J Med Chem 53: 4367-4378.

25. Holland JH (1975). "Adaptation in Natural and Artificial Systems". Ann Arbor, Univ of Michigan Press.

26. Holland JH (1998). "Emergence from Chaos to Order". Oxford University Press.

27. Janert PK (2010). "Gnuplot in Action. Understanding Data with Graphs". Greenwich, Connecticut: Manning Publications Co..

28. May RM (1976). Simple mathematical models with very complicated dynamics. Nature 261: 459-467. 
645 29. Dawkins R (1987) “Climbing Mount Improbable”, pp.236-245. London, Viking. 646 30. Dawkins R (1995) "River out of Eden", pp.6-11. London, Weidenfeld \& Nicolson.

647 31. Dawkins R (2004) “The Ancestor's Tale”, pp. 316-321, 346-354, 423-424. London,

648

649

650

651

652

653

654

655

656

657

658

659 Weidenfeld \& Nicolson.

32. Bradley JR, Johnson DR, Pober BR (2006). "Medical Genetics", $3^{\text {rd }}$ edition.. Oxford, Blackwell Publishing.

33. Schwartz JH (1999). "Sudden Origins. Fossils, Genes, and the Emergence of Species". New York: John Wiley and Sons, Inc..

34. Eldredge N, Gould SJ (1972). Punctuated equilibria: an alternative to phyletic gradualism. In "Models in Paleobiology"(edited by TMJ Schopf), Freeman, Cooper and Co, San Francisco, pp. 82-115.

35. Jones S (1993). "A Slower Kind of Bang” (review of E.O. Wilson, The Diversity of Life) London Review of Books, April, p.20.

36. Dennett DC (1995). “Darwin's Dangerous Idea”, pp.42-47. New York, Simon \& Schuster.

660 37. Arnold C (2013). The other you, New Scientist 12 January 2013, 31-34.

661

662

663

664

665

666

667

668

669

670

671

672

673

674

675

676

677

38. Kondrashov FA, Kondrashov AS (1999), Interactions among quantitative traits in the course of sympatric speciation. Nature 400: 351-354.

39. Savolainen V, Anstett M-C, Lexer C et al. (2006), Sympatric speciation in palms on an oceanic island. Nature 441: 210-213.

40. Bolnick DI (2006). Multispecies outcomes in a common model of sympatric speciation. J Theoret Biol 241: 734-744.

41. Nosil P (2008). Speciation with gene flow could be common. Molecular Ecology 17: 2103-21-6.

42. Malinsky M, Challis RJ, Tyers AM et al. (2015). Genome islands of speciation separate cichlid ecomorphs in an East African crater lake. Science 350: 1493-1498.

43. Wienberg J, Jauch A, Lüdecke HJ, Senger G, Horsthemke B, Claussen U, Cremer T, Arnold N, Lengauer C (1994). The origin of human chromosome 2 analyzed by comparative chromosome mapping with a DNA microlibrary. Chromosome Res 2: 404410.

44. Müller S, Wienberg J (2001). "Bar-coding” primate chromosomes: molecular cytogenetic screening for the ancestral hominoid karyotype. Hum Genet 109: 85-94.

678 


\section{Figure 1}

Normal operation of the SAC.

The vertical bars represent sister chromatids of germ cells in the second meiotic division, attached to each other by cohesin. The filled circles represent kinetochores, and the asterisks are spindle poles. Dashed lines indicate microtubules. 
A<smiles>[Tl]C1=CC2CC(=C1)C2</smiles>

B<smiles>CC12CC3C=CC(CC(C3)C1=O)C2</smiles>

no

C<smiles>[X]c1cc(C)cc(C)c1</smiles> 
Figure 2

Operation of the SAC following a chromosome fission.

The vertical bars represent sister chromatids of germ cells in the second meiotic division, attached to each other by cohesin. The filled circles represent kinetochores, and the asterisks are spindle poles. Dashed lines indicate microtubules. 


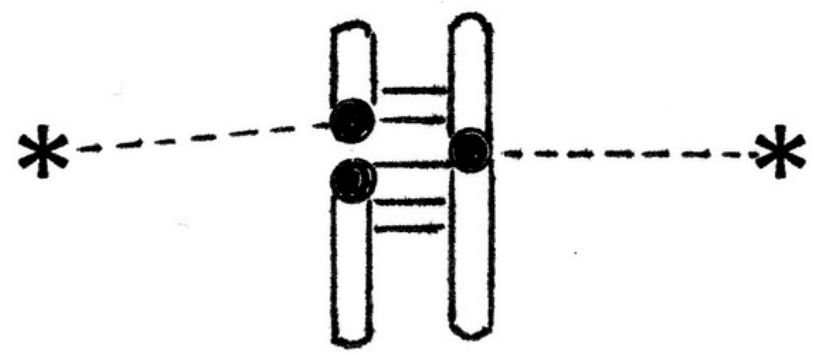

A

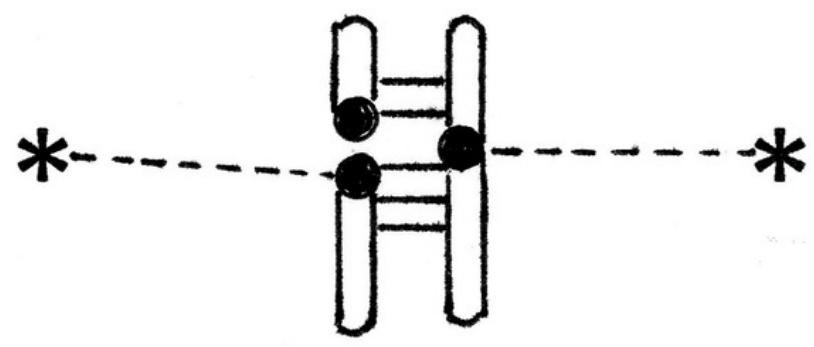

B

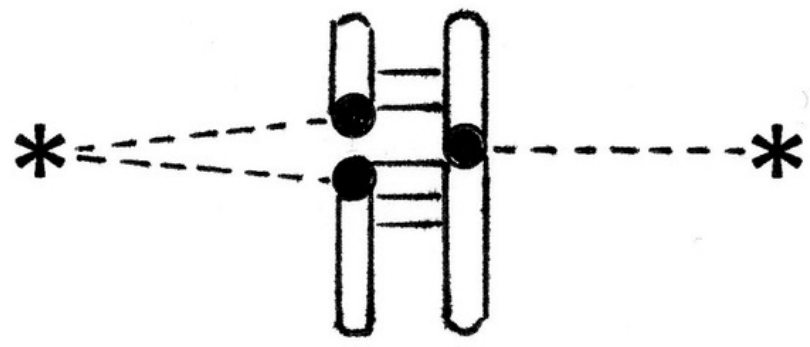<smiles>C1CCCCC1</smiles>

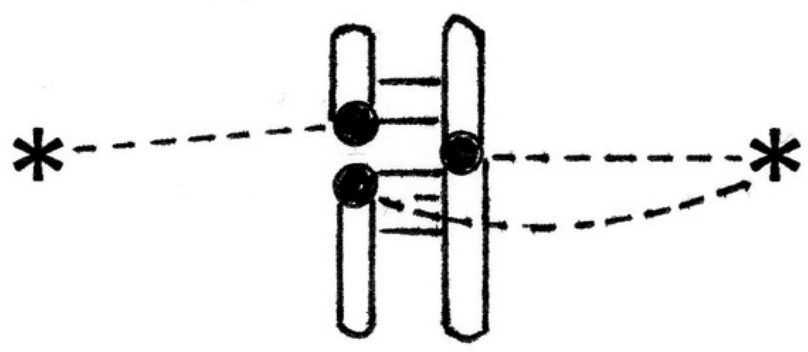<smiles>[2H]</smiles>

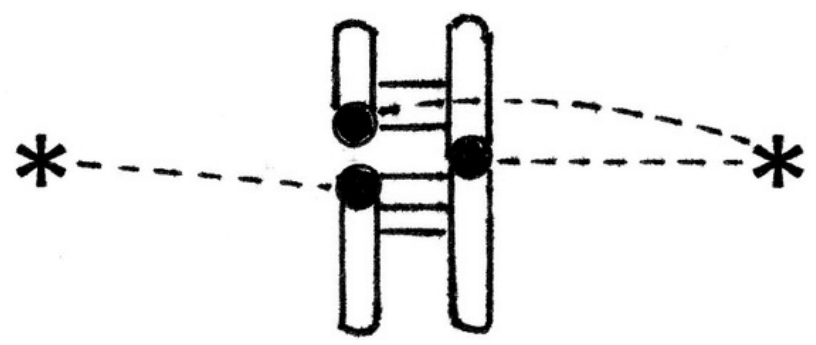


Figure 3

Fission Karyotypes

Karyotypes of gametes formed from meiosis of a cell in which karyotypic fission has occurred. The lower row of figures indicates the fraction of total gametes with the indicated karyotype.
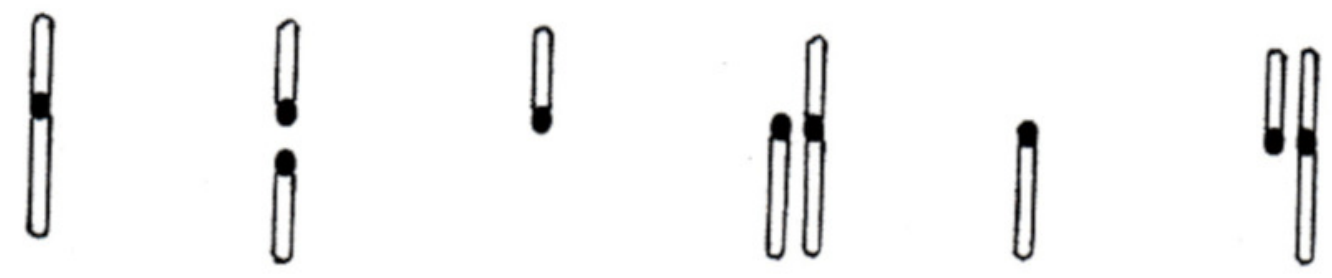
A
B
C
D
E
$\mathbf{F}$
$25 \%$
$25 \%$
$12.5 \%$
$12.5 \%$
$12.5 \%$
$12.5 \%$ 
Figure 4

Creation of Zygotes

Karyotypes of zygotes resulting from fertilisation of gametes of types I, II, IV and VI.

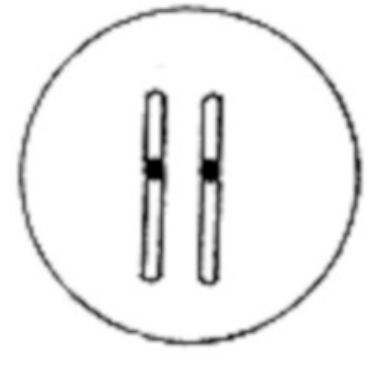

A

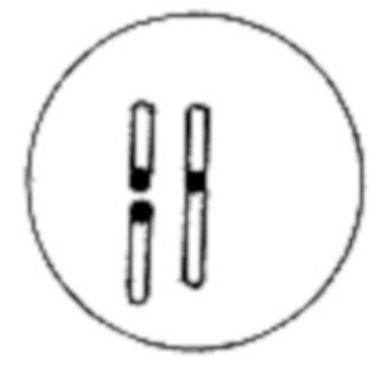

B

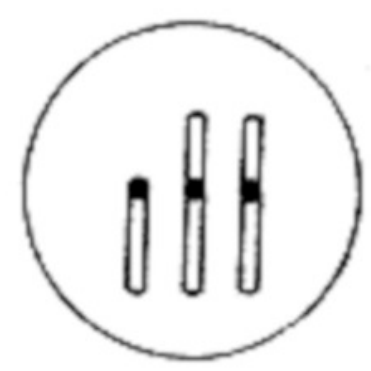

C

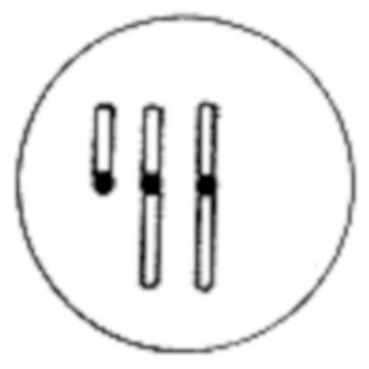

D 
Figure 5

\section{Growth Kinetics}

Growth of wild-type (Z1), chromosomal variant (Z3), and heterozygous (Z2) populations assuming a low initial population of heterozygotes, and exponential growth that ceases when the total population reaches the carrying capacity of the habitat.

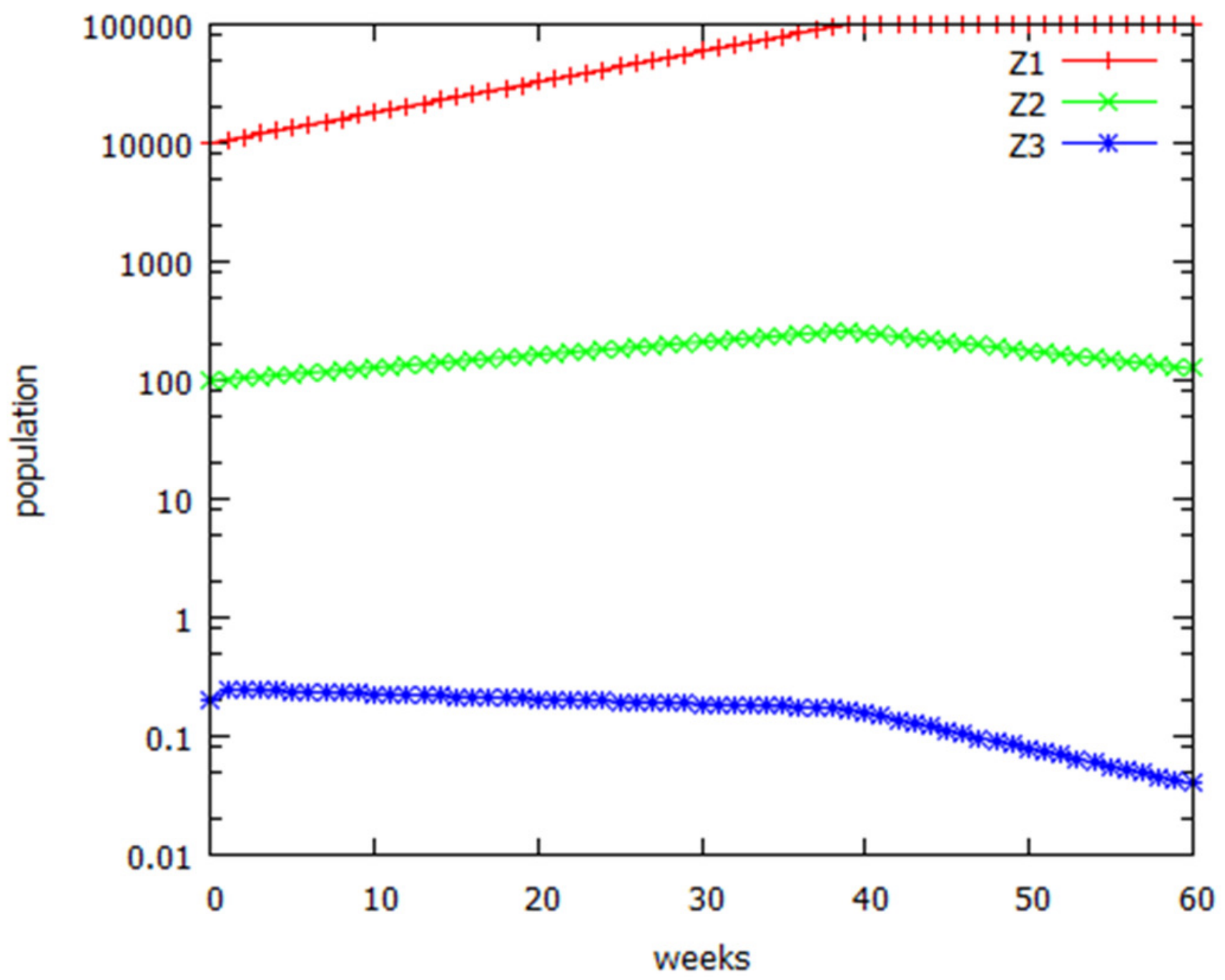


Figure 6

Logistic Growth

Modelling wild-type (Z1), variant (Z3), and heterozygous (Z2) populations using the discrete logistic growth equation.

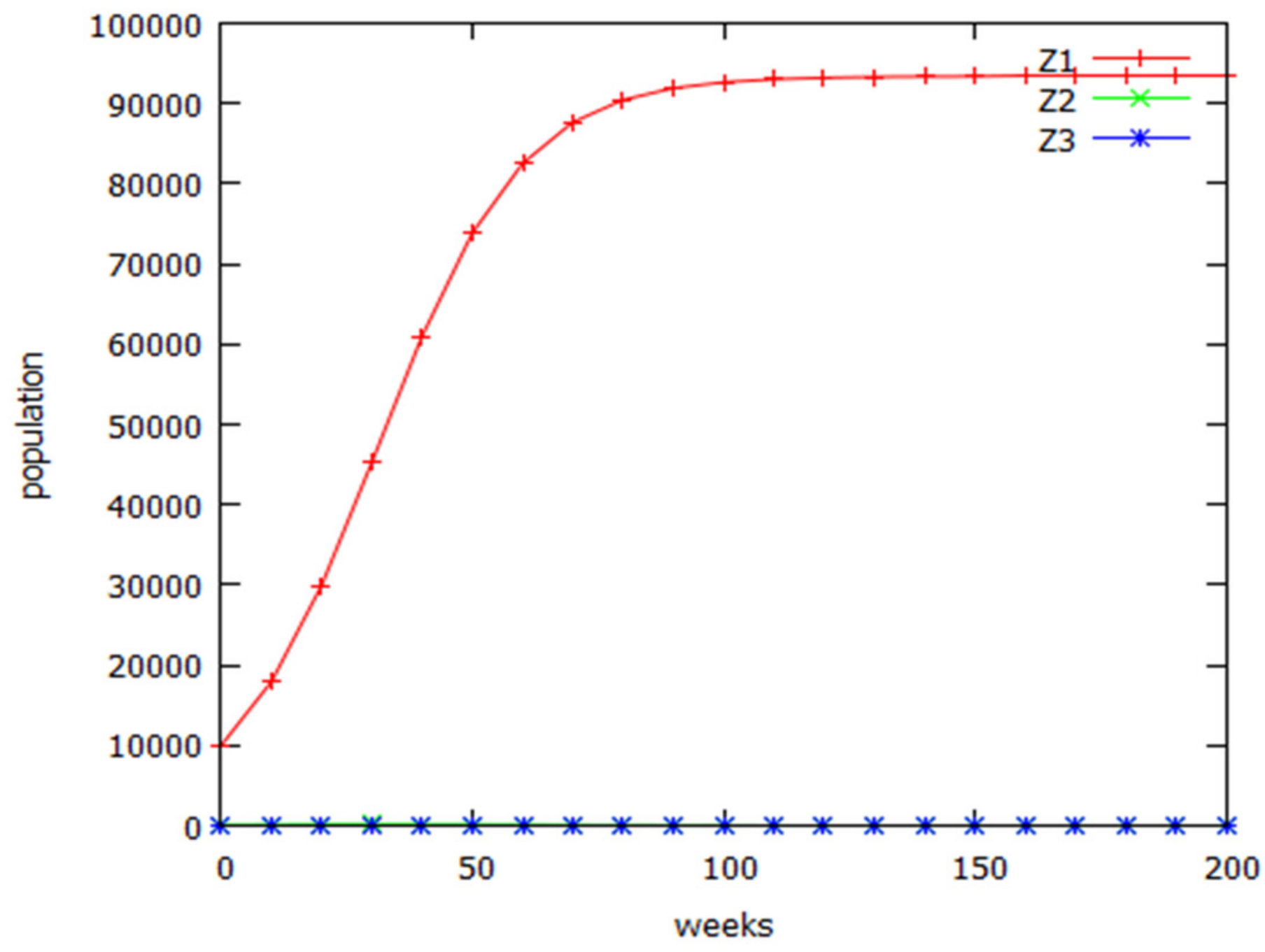




\section{Figure 7}

\section{Peripatric Speciation}

Peripatric speciation when the wild-type population is very poorly adapted to the second ecological niche (data of table 3, simulation 3). Z1, Z2, and Z3 are wild-type, heterozygote, and variant populations, respectively, in niche 1 , and Z4, Z5, and Z6 are wild-type, heterozygote, and variant populations, respectively, in niche 2 . Upper panel: niche 1; lower panel: niche 2 . 

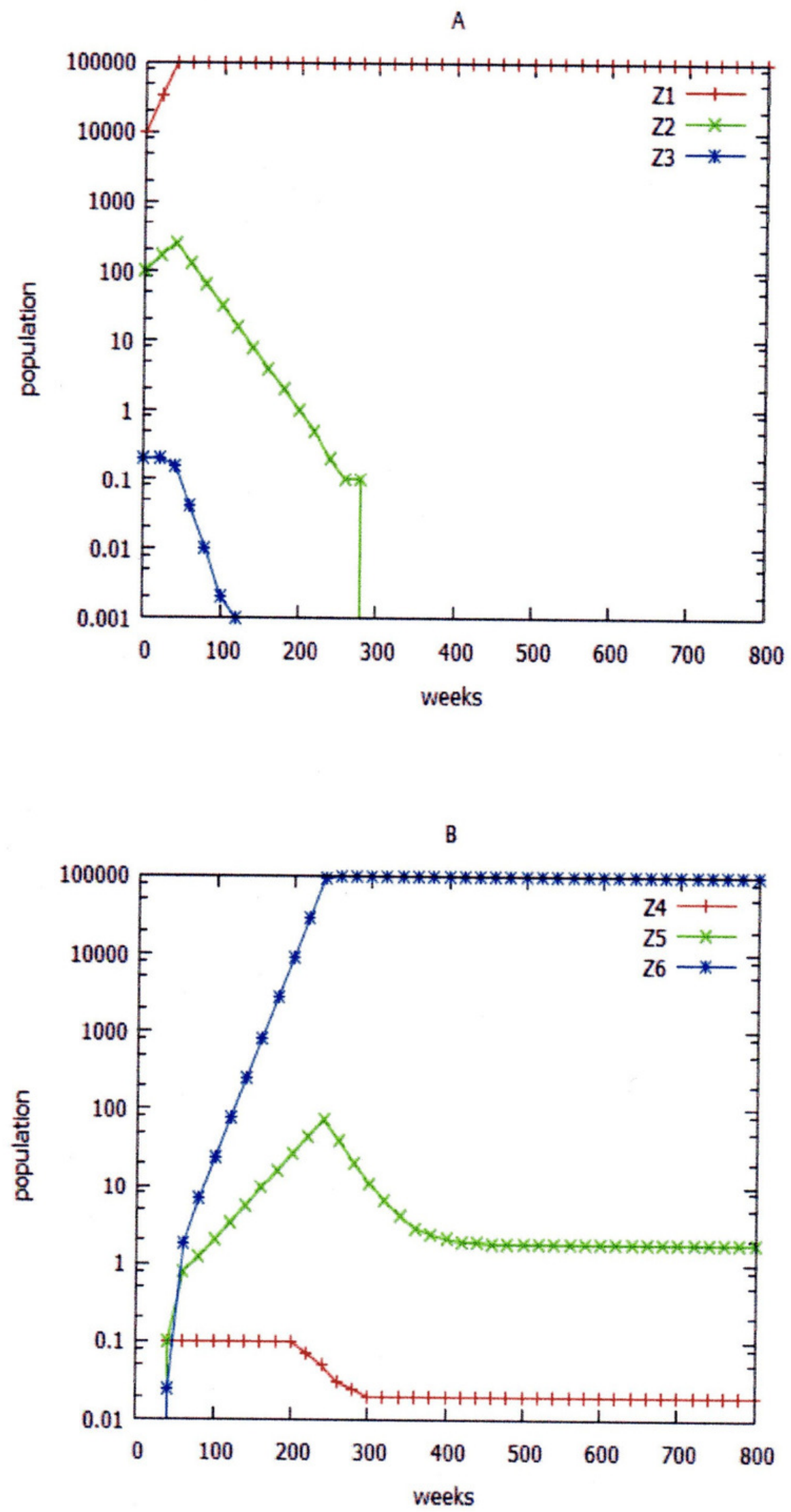
Figure 8

Geography and Colonization

Geographical isolation followed by re-colonization. Populations of wild-type (Z4), heterozygotes (Z5) and variant homozygotes (Z6) are shown in niche 2. Migration from niche 1 to niche 2 was assumed to occur until week 350, after which it stops, but resumes at 800 weeks.

Niche 2

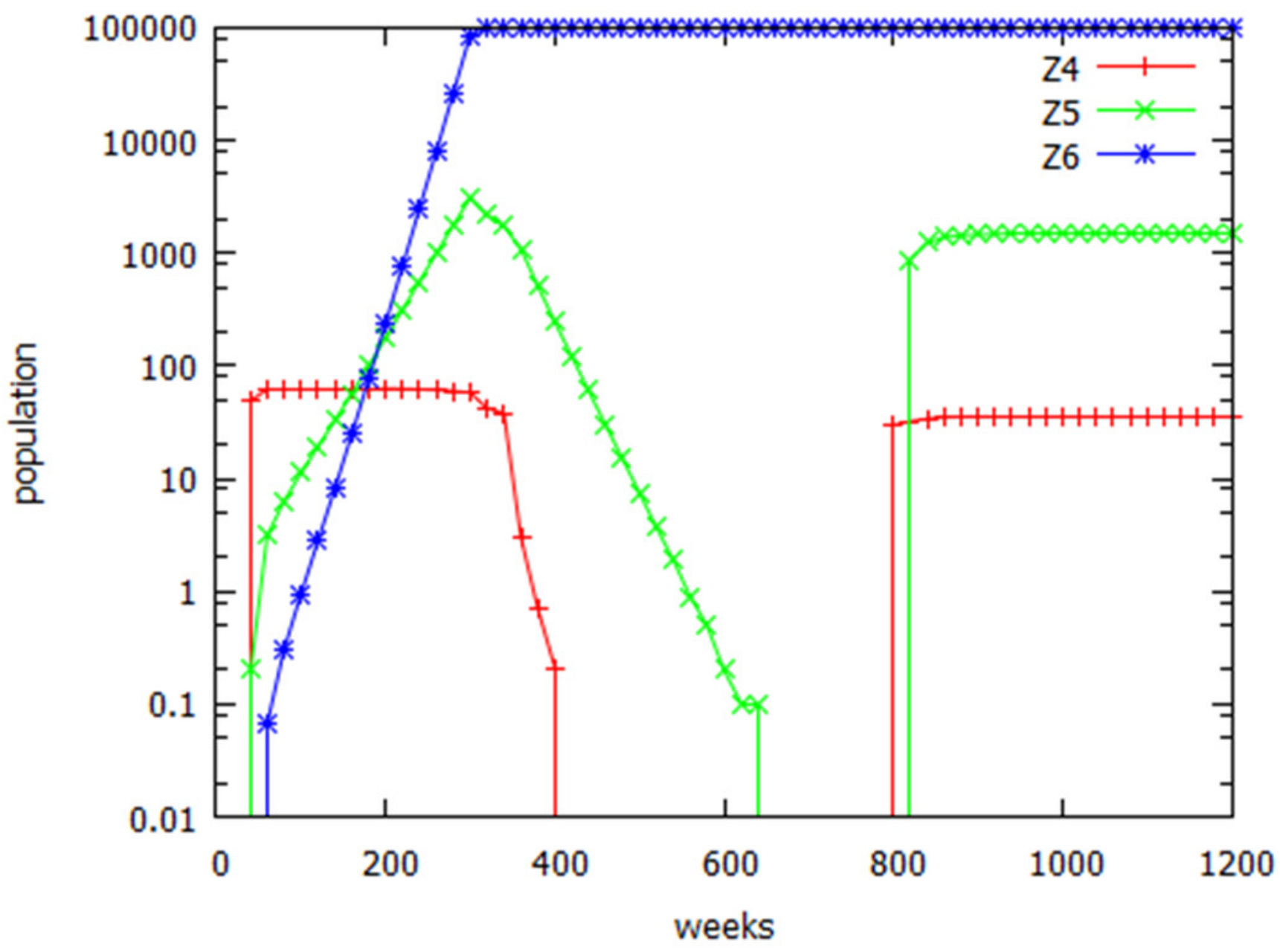




\section{Table $\mathbf{1}$ (on next page)}

Evolutionary dynamics of chromosomal variants in a single ecological niche

Geographical isolation followed by re-colonization. Populations of wild-type (Z4), heterozygotes (Z5) and variant homozygotes (Z6) are shown in niche 2. Migration from niche 1 to niche 2 was assumed to occur until week 350 , after which it stops, but resumes at 800 weeks. 
Table 1

\section{Evolutionary dynamics of chromosomal variants in a single ecological niche}

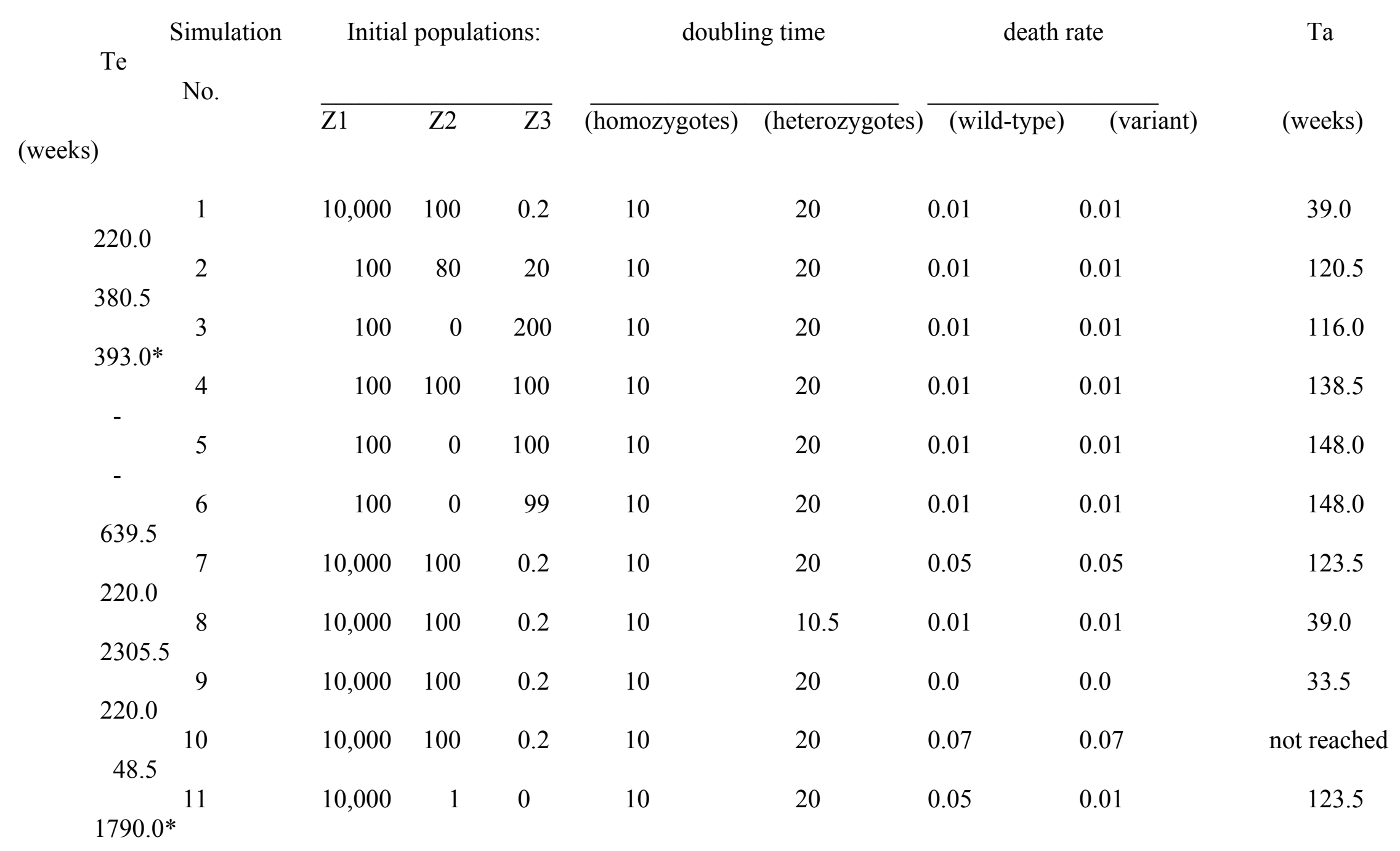




$\begin{array}{cccccc} & 12 & 10,000 & 1 & 0 & 10 \\ 273.5 & 13 & 10,000 & 1 & 0 & 10 \\ 7360.0 * & 14 & 10,000 & 1 & 0 & 10\end{array}$

20

20

20
0.05

0.05

0.05
0.01

0.017

0.015
123.5

Simulations calculated populations of wild-type homozygotes (Z1), heterozygotes (Z2), and variant homozygotes (Z3). Growth was assumed to be exponential until the carrying capacity of the habitat was reached, after which the total population remained constant. Doubling times in weeks; death rate, fraction of population dying per week. Where the death rate of wild-type and variant homozygotes differs, the death rate for heterozygotes will be that of the chromosomal variants for dominant mutations, and of the wild-type for recessive mutations. Ta, time to carrying capacity; Te, time to extinction of variants (except for simulations 3 and 11 , where the wild-type becomes extinct).

* Extinction of wild-type 


\section{Table 2 (on next page)}

Effect of different growth curves on evolutionary dynamics of wild-type and chromosomal variant populations

Growth of wild-type and chromosomal-variant organisms was modelled in a single niche, and times to extinction of the variant were modelled using different growth equations.

Growth parameters were adjusted to give comparable time to $85 \%\left(\mathrm{~T}_{0.85}\right)$ of asymptote for all growth curves.

* Doubling time (homozygotes) 11.6 weeks, (heterozygotes) 23.2 weeks; death rate (all populations) 0.07/week.

* Doubling time (homozygotes) 1.14 weeks, (heterozygotes) 2.28 weeks.

I Doubling time (homozygotes) 5.9 weeks, (heterozygotes) 11.8 weeks 
1

2

3

4

5

6

7

8

9

10

11

12

13

14

15

16

17

18

19 20

21

22

23

24

25

26

Table 2

\section{Effect of different growth curves on evolutionary dynamics of wild-type and chromosomal variant populations}

Growth curve $\quad \mathrm{T}_{0.85} \quad$ Te (weeks)

Exponential * $\quad 43.0 \quad 255.0$

Gompertzian $\underset{*}{*} \quad 43.0 \quad 1951.5$

Logistic $9 \quad 43.0 \quad 133.5$

Growth of wild-type and chromosomal-variant organisms was modelled in a single niche, and times to extinction of the variant were modelled using different growth equations.

Growth parameters were adjusted to give comparable time to $85 \%\left(\mathrm{~T}_{0.85}\right)$ of asymptote for all growth curves.

* Doubling time (homozygotes) 11.6 weeks, (heterozygotes) 23.2 weeks; death rate (all populations) $0.07 /$ week.

* Doubling time (homozygotes) 1.14 weeks, (heterozygotes) 2.28 weeks.

ๆ Doubling time (homozygotes) 5.9 weeks, (heterozygotes) 11.8 weeks 


\section{Table 3(on next page)}

Colonisation of an adjoining niche by organisms originating from a single heterozygote in niche 1

Population dynamics of wild-type and variant organisms whose range spans covers two adjacent or overlapping niches. Z1, wild-type homozygotes in niche1; Z2, heterozygotes in niche 1, Z3, variant homozygotes in niche 1, Z4, wild-type homozygotes in niche 2; Z5, heterozygotes in niche 2, Z6, variant homozygotes in niche 2.

Doubling times: Z1, Z3, Z4 and Z6: 10 weeks Z2 and Z5: 20 weeks (except simulations 7 and 8 ) 40 weeks (simulations 7 and 8)

Death rates: 0.01 for Z1, Z2, Z3 and Z6; Z5, 0.01 except for simulation 4, where it is 0.99; Z4, 0.99, except for simulation 1 , where it is 0.01 
Table 3

\section{Colonisation of an adjoining niche by organisms originating from a single heterozygote in niche 1}

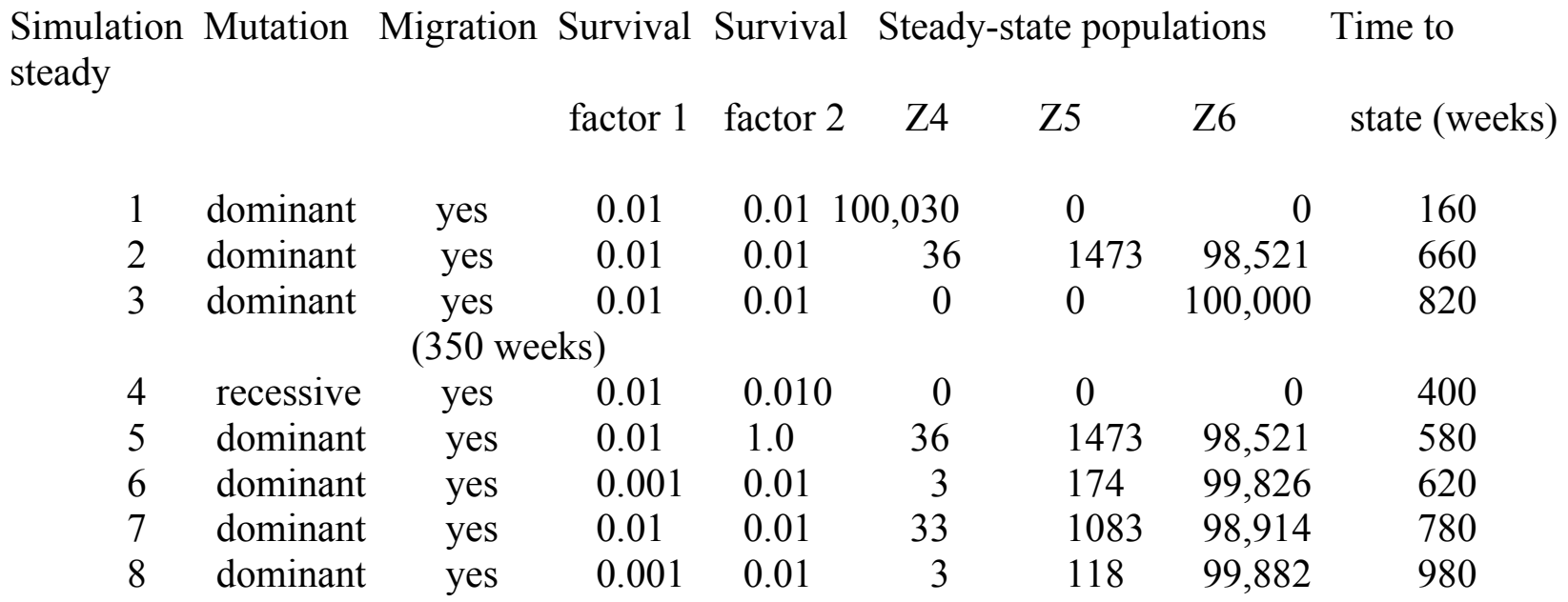

Population dynamics of wild-type and variant organisms whose range spans covers two adjacent or overlapping niches. Z1, wild-type homozygotes in niche1; Z2, heterozygotes in niche 1, Z3, variant homozygotes in niche $1, \mathrm{Z} 4$, wild-type homozygotes in niche 2 ; Z5, heterozygotes in niche 2, Z6, variant homozygotes in niche 2 .

Doubling times: Z1, Z3, Z4 and Z6: 10 weeks

Z2 and Z5: 20 weeks (except simulations 7 and 8 ) 40 weeks (simulations 7 and 8 )

Death rates: 0.01 for Z1, Z2, Z3 and Z6; Z5, 0.01 except for simulation 4, where it is 0.99; $\mathrm{Z} 4,0.99$, except for simulation 1 , where it is 0.01 


\section{Table 4 (on next page)}

Population Dynamics in Two Adjoining Ecological Niches Modelled by a Discrete Logistic Growth Curve

Population dynamics of wild-type and variant organisms whose range spans covers two adjacent or overlapping niches. Z1, wild-type homozygotes in niche1; Z2, heterozygotes in niche 1, Z3, variant homozygotes in niche 1, Z4, wild-type homozygotes in niche 2; Z5, heterozygotes in niche 2, Z6, variant homozygotes in niche 2 .

Doubling times: Z1, Z3, Z4 and Z6: 10 weeks. Z2 and Z5: 20 weeks

Initial population counts: Z1, 10,000; Z2, 1; Z3, 0; Z4, 0 (except for simulations 6 and 7); Z5; 0, Z6, 0. Simulations 6 and 7, Z4 = 1,000 
Table 4

Population Dynamics in Two Adjoining Ecological Niches Modelled by a Discrete Logistic Growth Curve

\begin{tabular}{|c|c|c|c|c|c|c|c|c|c|c|c|c|c|c|c|c|}
\hline \multirow{2}{*}{\multicolumn{2}{|c|}{$\begin{array}{l}\text { steady } \\
\text { (weeks) }\end{array}$}} & \multirow[t]{2}{*}{ Mutation } & \multicolumn{6}{|c|}{ Death rates } & \multirow{2}{*}{$\begin{array}{l}\text { Reverse } \\
\text { migration }\end{array}$} & \multirow{2}{*}{$\begin{array}{l}\text { Survival } \\
\text { factor } 1\end{array}$} & \multirow{2}{*}{$\begin{array}{l}\text { Survival } \\
\text { factor } 2\end{array}$} & \multicolumn{4}{|c|}{ Steady-state populations } & \multirow{2}{*}{$\begin{array}{l}\text { Time to } \\
\text { state }\end{array}$} \\
\hline & & & $\mathrm{Z} 1$ & $\mathrm{Z} 2$ & $\mathrm{Z3}$ & $\mathrm{Z} 4$ & $\mathrm{Z} 5$ & Z6 & & & & $\mathrm{Z} 2$ & $\mathrm{Z} 4$ & $\mathrm{Z5}$ & Z6 & \\
\hline & 1 & dominant & 0.001 & 0.001 & 0.001 & 0.99 & 0.001 & 0.001 & no & $1.0 \mathrm{e}-6$ & 1.0 & 0 & 0.1 & 0.6 & 92110 & 4 \\
\hline & 2 & dominant & 0.001 & 0.001 & 0.001 & 0.99 & 0.001 & 0.001 & yes & $1.0 \mathrm{e}-6$ & 1.0 & 1 & 0.1 & 133 & 92051 & 4 \\
\hline & 3 & dominant & 0.001 & 0.001 & 0.001 & 0.99 & 0.001 & 0.001 & yes & 0 & 1.0 & 4 & 0 & 133 & 92051 & 4 \\
\hline & 4 & dominant & 0.010 & 0.010 & 0.010 & 0.99 & 0.010 & 0.010 & yes & $1.0 \mathrm{e}-6$ & 1.0 & 3 & 0.1 & 87 & 80231 & 42 \\
\hline & 5 & recessive & 0.010 & 0.010 & 0.010 & 0.99 & 0.99 & 0.010 & no & $1.0 \mathrm{e}-6$ & 1.0 & 3 & 0.1 & 2.6 & 80273 & 4 \\
\hline & 6 & dominant & 0.060 & 0.060 & 0.060 & 0.99 & 0.060 & 0.060 & yes & $1.0 \mathrm{e}-6$ & 1.0 & 0.1 & 0 & 0.2 & 10363 & 208 \\
\hline & 7 & dominant & 0.070 & 0.070 & 0.070 & 0.99 & 0.070 & 0.060 & yes & $1.0 \mathrm{e}-6$ & 1.0 & 0 & 0 & 0 & 0 & 295 \\
\hline
\end{tabular}

Population dynamics of wild-type and variant organisms whose range spans covers two adjacent or overlapping niches. Z1, wild-type homozygotes in niche1; Z2, heterozygotes in niche 1, Z3, variant homozygotes in niche 1, Z4, wild-type homozygotes in niche 2; Z5, heterozygotes in niche 2, Z6, variant homozygotes in niche 2 .

Doubling times: Z1, Z3, Z4 and Z6: 10 weeks. Z2 and Z5: 20 weeks

Initial population counts: Z1, 10,000; Z2, 1; Z3, 0; Z4, 0 (except for simulations 6 and 7); Z5; 0, Z6, 0. Simulations 6 and 7, Z4 =1,000 